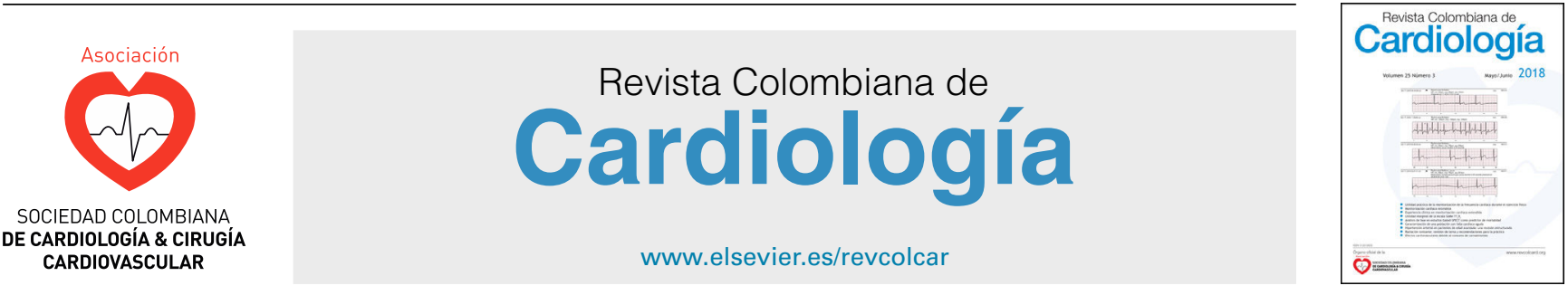

CARDIOLOGÍA DEL ADULTO - ARTÍCULO ORIGINAL

\title{
Caracterización clínica y demográfica de pacientes adultos sometidos a valoración de la presión arterial con monitorización ambulatoria de la presión arterial. Registro EPEDMAPA
}

\author{
Álvaro Herrera Escandón ${ }^{a}$, Luis Fernando Sánchez Solanilla ${ }^{b}$, \\ José Alejandro Ramírez-Penuela ${ }^{c}$ y Andrés Felipe Buitrago Sandoval ${ }^{b, c, d, e, f, *}$
}

a Facultad de Salud, Universidad del Valle, Cali, Colombia

b Universidad El Bosque, Bogotá, Colombia

c Facultad de Medicina, Universidad de los Andes, Bogotá, Colombia

d Sección de Cardiología, Fundación Santafé de Bogotá, Bogotá, Colombia

e Departamento de Cuidado Crítico, Fundación Santafé de Bogotá, Bogotá, Colombia

f Universidad del Rosario, Bogotá, Colombia

Recibido el 4 de julio de 2018; aceptado el 22 de julio de 2019

Disponible en Internet el 25 de noviembre de 2019

\section{PALABRAS CLAVE \\ Hipertensión; Monitorización ambulatoria de presión arterial; Diagnóstico; Registro}

\begin{abstract}
Resumen
Introducción: la hipertensión arterial es un problema de salud pública que aumenta la mortalidad en todos los escenarios clínicos, pero es, además, el principal factor de riesgo modificable. Es una enfermedad altamente prevalente; cerca de un cuarto de la población del mundo la padece. Pocos pacientes la conocen y pocos están tratados de manera óptima.

Objetivo: evaluar las características de pacientes a quienes se les realizó una monitorización ambulatoria de la presión arterial en un Hospital Universitario, con miras a describir el perfil clínico y demográfico.

Métodos: estudio descriptivo retrospectivo, llevado a cabo en pacientes sometidos a monitorización ambulatoria de la presión arterial de 24 horas durante los meses de octubre y noviembre de 2015.

Resultados: se confirmó hipertensión (de reciente diagnóstico o conocida) en el $75 \%$ de los estudios realizados. Se descartó hipertensión arterial en el 31\% de los pacientes previamente clasificados como hipertensos. El $61 \%$ de los pacientes que se encontraban bajo tratamiento estaban bien controlados, la mayoría de ellos con un solo medicamento, principalmente antagonistas del receptor de angiotensina II. El patrón circadiano más prevalente en esta cohorte de pacientes fue el dipper (48\%) seguido por el patrón de non-dipper (29\%).
\end{abstract}

\footnotetext{
* Autor para correspondencia.

Correo electrónico: abuitrag@uniandes.edu.co (A.F. Buitrago Sandoval).
} 


\section{KEYWORDS}

Hypertension; Ambulatory blood pressure monitoring; Diagnosis; Registry
Conclusiones: el monitorización ambulatoria de la presión arterial permite evaluar con exactitud el estado de la presión arterial de los pacientes con sospecha de hipertensión arterial. Esto aclara si los pacientes son realmente normotensos o hipertensos y discrimina las condiciones de la bata blanca y la hipertensión enmascarada, con lo cual se evitan tratamientos innecesarios y se favorece un mejor control de la presión arterial.

(c) 2019 Publicado por Elsevier España, S.L.U. en nombre de Sociedad Colombiana de Cardiología y Cirugía Cardiovascular. Este es un artículo Open Access bajo la licencia CC BY-NC-ND (http:// creativecommons.org/licenses/by-nc-nd/4.0/).

\section{Clinical and demographic profile of adult patients subjected to an assessment of arterial hypertension with an ambulatory blood pressure monitoring device. EPEDMAPA Registry}

\begin{abstract}
Introduction: Arterial hypertension is a public health problem that increases mortality in all clinical situations. It is also the main modifiable risk factor. It is a highly prevalent condition that is suffered by around $25 \%$ of the world population. Few patients are aware of it, and few receive the optimum treatment.

Objective: To evaluate the characteristics of the patients on whom ambulatory blood pressure monitoring was carried out in a University Hospital, with a view to describing the clinical and demographic profile.

Methods: A descriptive retrospective study was conducted on patients subjected to 24-hour ambulatory blood pressure monitoring, during the months of October and December 2015.

Results: Hypertension (recently diagnosed or known) was confirmed in $75 \%$ of the studies performed. Arterial hypertension was ruled out in $31 \%$ of the patients previously classified as hypertensive. Of the patients that were receiving treatment, $61 \%$ were well-controlled, with the majority of them with a single drug, mainly an angiotensin II receptor agonist. The dipper was most prevalent circadian pattern, with $48 \%$, followed by the non-dipper pattern in $29 \%$.

Conclusions: Ambulatory blood pressure monitoring helps in the evaluation of the blood pressure status accurately in patients with a suspicion of arterial hypertension. This clarifies whether the patients are really normotensive or hypertensive and discriminates between the "white coat" and masked hypertension conditions. This avoids unnecessary treatments and favours a better control of the blood pressure.

(c) 2019 Published by Elsevier España, S.L.U. on behalf of Sociedad Colombiana de Cardiología y Cirugía Cardiovascular. This is an open access article under the CC BY-NC-ND license (http:// creativecommons.org/licenses/by-nc-nd/4.0/).
\end{abstract}

\section{Introducción}

La hipertensión arterial es considerada un grave problema de salud pública, de impacto significativo en muchos escenarios clínicos, al que se le atribuye un riesgo mayor de mortalidad general y severidad de cualquier enfermedad. Es, además, el principal factor de riesgo modificable para padecer eventos cerebrovasculares, cardiovasculares y enfermedad renal ${ }^{1}$.

Esta enfermedad afecta cerca de 1 billón de personas en el mundo; su prevalencia en hombres es del $29,2 \%$ y en mujeres del $24,8 \%$, porcentajes que varían de acuerdo con el grupo poblacional y otros factores socio-demográficos; ésta puede oscilar entre el 20 al $50 \%$ en países desarrollados y ser de mayor prevalencia en poblaciones de afrodescendientes ${ }^{2}$.

De acuerdo con datos del estudio de enfermedades crónicas - ENFREC II - realizado en Colombia, la prevalencia estimaba un $12,3 \%$ de personas con hipertensión arterial para el año $1999^{3}$. Posteriormente para Bogotá en el año 2005 , el estudio CARMELA reconoció un $13,5 \%$ de la población con esta misma condición, de los cuales un $68,8 \%$ tenían conciencia de la enfermedad, $55 \%$ se encontraban bajo tratamiento y solo el $30 \%$ de estos estaban controlados ${ }^{4}$.

La identificación pronta de los pacientes con hipertensión arterial es crucial para el inicio del tratamiento y la prevención de complicaciones.

Al día de hoy la medición manual con esfigmomanómetro y estetoscopio es el método de elección, al ser una herramienta de bajo costo y fácilmente disponible, pero con serias limitaciones en comparación con otros métodos que utilizan formas automatizadas.

Entre estos métodos automatizados se encuentra la monitorización ambulatoria de la presión arterial de 24 horas, considerada como un sistema de alta calidad que aborda la enfermedad con relación al entorno del paciente, suprime el estrés de bata blanca y proporciona datos adicionales hemodinámicos de gran utilidad. Sus principales beneficios comparados con el método manual radican en reducir errores y proveer mediciones más precisas y con rangos que 
suelen ser menores a la toma manual ${ }^{5}$. Este estudio tiene variables adicionales a la toma de presión única que permiten estimar otros aspectos hemodinámicos y de correlación con la eficacia del tratamiento, los cuales no se pueden determinar con la automedición en casa o medición en el consultorio.

Con el estudio EPEDMAPA se pretende evaluar las características de pacientes a quienes se les realizó una monitorización ambulatoria de la presión arterial en un Hospital Universitario, con el fin de describir el perfil clínico y demográfico.

\section{Metodología}

Se hizo un estudio descriptivo de carácter retrospectivo, en personas sometidas a una monitorización ambulatoria de presión arterial ( 24 horas) en el período comprendido entre octubre y noviembre de 2015 en un hospital universitario.

Se incluyeron adultos mayores de 18 años, los cuales fueron programados para una monitorización ambulatoria de la presión arterial como estudio diagnóstico de hipertensión arterial de acuerdo con el criterio del médico tratante o para una monitorización ambulatoria de la presión arterial para evaluación del control de tratamiento de la hipertensión arterial.

Fueron excluídos los casos de incapacidad para tolerar la toma de presión arterial (retraso mental, decisión del paciente, ausencia de extremidades superiores o intolerancia al dispositivo). Los estudios de monitorización ambulatoria de presión arterial que no cumplieron con los criterios de calidad adecuada en su toma no se registraron para su interpretación (al menos el 70\% o más de las tomas de presión arterial esperadas debían estar registradas).

Se construyó una base de datos de los pacientes con estudio de monitorización ambulatoria de la presión arterial evaluados. Se codificaron las variables demográficas, farmacológicas, hemodinámicas y los resultados de la monitorización. El análisis estadístico se hizo en Microsoft Excel para MAC versión 14.5.9, mediante el diseño de una hoja de cálculo en la que se inscribieron las variables de interés. Se calcularon promedios, frecuencias y gráficas para la interpretación de los resultados.

\section{Resultados}

Un total de 115 pacientes a quienes se les realizó una monitorización ambulatoria de presión arterial cumplieron con los criterios de calidad de la toma y fueron incluidos en el análisis de este estudio.

La edad promedio de los pacientes fue de 56,9 años (desviación estándar: 3,5 años). La división por sexo mostró una edad promedio en hombres de 55,6 años y en mujeres de 58,5 años. El peso promedio de los pacientes fue de $73,2 \mathrm{~kg}$ (DS: $13,4 \mathrm{~kg}$ ). La estatura promedio fue de $167,1 \mathrm{~cm}$ (DS: $5,6 \mathrm{~cm}$ ). El índice de masa corporal (IMC) promedio fue de $26 \mathrm{~kg} / \mathrm{m}^{2}$. El promedio de pacientes con $I M C \geq 25 \mathrm{~kg} / \mathrm{m}^{2}$ fue de $57,3 \%$.

El porcentaje total de los pacientes incluidos en el estudio que fueron clasificados con diagnóstico de hipertensión arterial sistémica se describe en la figura 1. Cabe resaltar este $31 \%$ de pacientes que previamente habían sido

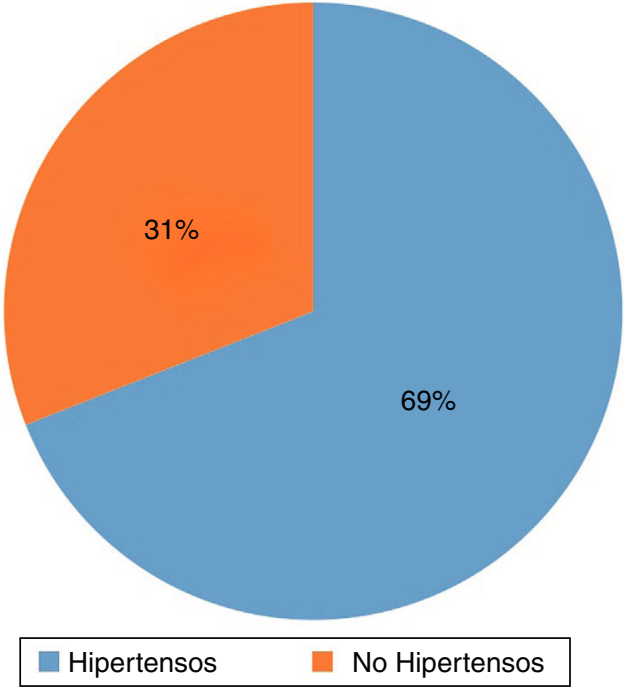

Figura 1 Porcentaje de pacientes hipertensos del total de la muestra analizada.

Tabla 1 Comorbilidades asociadas

\begin{tabular}{ll}
\hline Variable & Porcentaje \\
\hline Diabetes mellitus & $8,6 \%$ \\
Dislipidemia & $39,1 \%$ \\
Tabaquismo & $29,5 \%$ \\
Obesidad & $17,3 \%$ \\
Evento cerebrovascular previo & $3,4 \%$ \\
Estrés & $40 \%$ \\
Sedentarismo & $75,6 \%$ \\
Consumo de alcohol & $6,9 \%$ \\
\hline
\end{tabular}

clasificados como hipertensos, pero cuyo reporte de monitorización ambulatoria de la presión arterial excluyó la entidad.

Así mismo, se encontró un interesante porcentaje de comorbilidades en el que se resaltan el sedentarismo, perfil subjetivo de estrés emocional, tabaquismo y dislipidemia. Las comorbilidades asociadas se describen en la tabla 1.

Uno de los resultados más relevantes de este estudio fue la caracterización de los pacientes hipertensos. La hipertensión arterial nocturna, que según lo descrito en la literatura parece predecir mejor el riesgo cardiovascular que la presión arterial diurna ${ }^{6}$, se encontró en el $32 \%$ de los pacientes hipertensos. Por otro lado, el porcentaje de hipertensos conocidos adecuadamente controlados correspondió al $61 \%$ de la población con la enfermedad (tabla 2).

Al evaluar la cantidad de medicamentos antihipertensivos que reciben estos pacientes la mayoría (65\%) estaban siendo tratados con un solo medicamento (fig. 2). Al analizar la terapia antihipertensiva de acuerdo con grupos farmacológicos, la mayoría de los pacientes recibía antagonistas del receptor de angiotensina II y ningún paciente recibía antagonistas mineralocorticoides (tabla 3).

En cuanto a las variables hemodinámicas encontradas (tabla 4), cabe resaltar que el porcentaje de cargas de presión arterial (porcentaje de lecturas sistólicas y diastólicas que se encuentran por encima de los rangos de normalidad) que se obtuvo para el periodo diurno fue de $30,6 \%$ para las 
Tabla 2 Caracterización de la población hipertensa

\begin{tabular}{lll}
\hline Variable & $\mathrm{n}$ & Porcentaje \\
\hline $\begin{array}{l}\text { Total de pacientes con hipertensión } \\
\text { arterial }\end{array}$ & 79 & $100 \%$ \\
Pacientes con hipertensión conocida & 52 & $65 \%$ \\
Pacientes con hipertensión de & 27 & $35 \%$ \\
reciente diagnóstico & & \\
Hipertensos sistólicos aislados & 20 & $25 \%$ \\
Hipertensos diastólicos aislados & 27 & $35 \%$ \\
Hipertensión de predominio diurno & 26 & $32 \%$ \\
Hipertensión de predominio nocturno & 29 & $36 \%$ \\
Hipertensos conocidos controlados & 32 & $61 \%$ \\
\hline
\end{tabular}

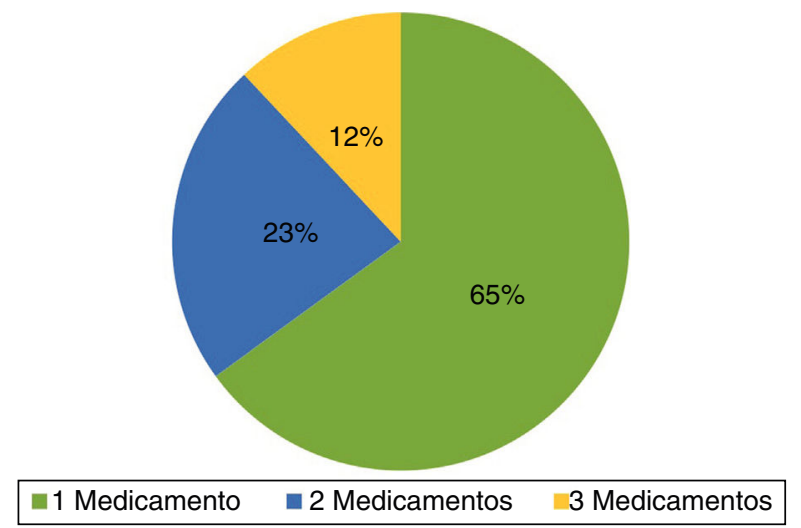

Figura 2 Porcentaje de pacientes de acuerdo con la cantidad de medicamentos que reciben.

Tabla 3 Descripción del manejo farmacológico antihipertensivo

\begin{tabular}{llc}
\hline Variable & $\mathrm{n}$ & $\%$ \\
\hline $\begin{array}{l}\text { Inhibidores de la enzima convertidora } \\
\text { de angiotensina }\end{array}$ & 7 & $6 \%$ \\
$\begin{array}{l}\text { Bloqueadores del receptor de } \\
\text { angiotensina II }\end{array}$ & 33 & $29 \%$ \\
Calcio-antagonistas & 15 & $13 \%$ \\
Diuréticos & 5 & $4 \%$ \\
Antagonistas mineralocorticoides & 0 & $0 \%$ \\
Betabloqueadores & 15 & $13 \%$ \\
Vasodilatadores & 0 & $0 \%$ \\
\hline
\end{tabular}

lecturas sistólicas y $38,6 \%$ para las diastólicas, mientras que para la noche el porcentaje de cargas sistólicas fue de $28,7 \%$ y el de diastólicas de $44 \%$.

En la figura 3 se describe el patrón circadiano de la presión arterial. Se observa un alto porcentaje (32\%) de pacientes non-dipper y reverse dipper.

\section{Discusión}

Hasta el momento los datos acerca del comportamiento de la hipertensión arterial en Colombia son escasos. Las poblaciones descritas previamente han sido evaluadas a través de la toma de presión arterial en el consultorio, con
Tabla 4 Variables hemodinámicas de la monitorización ambulatoria de la presión arterial

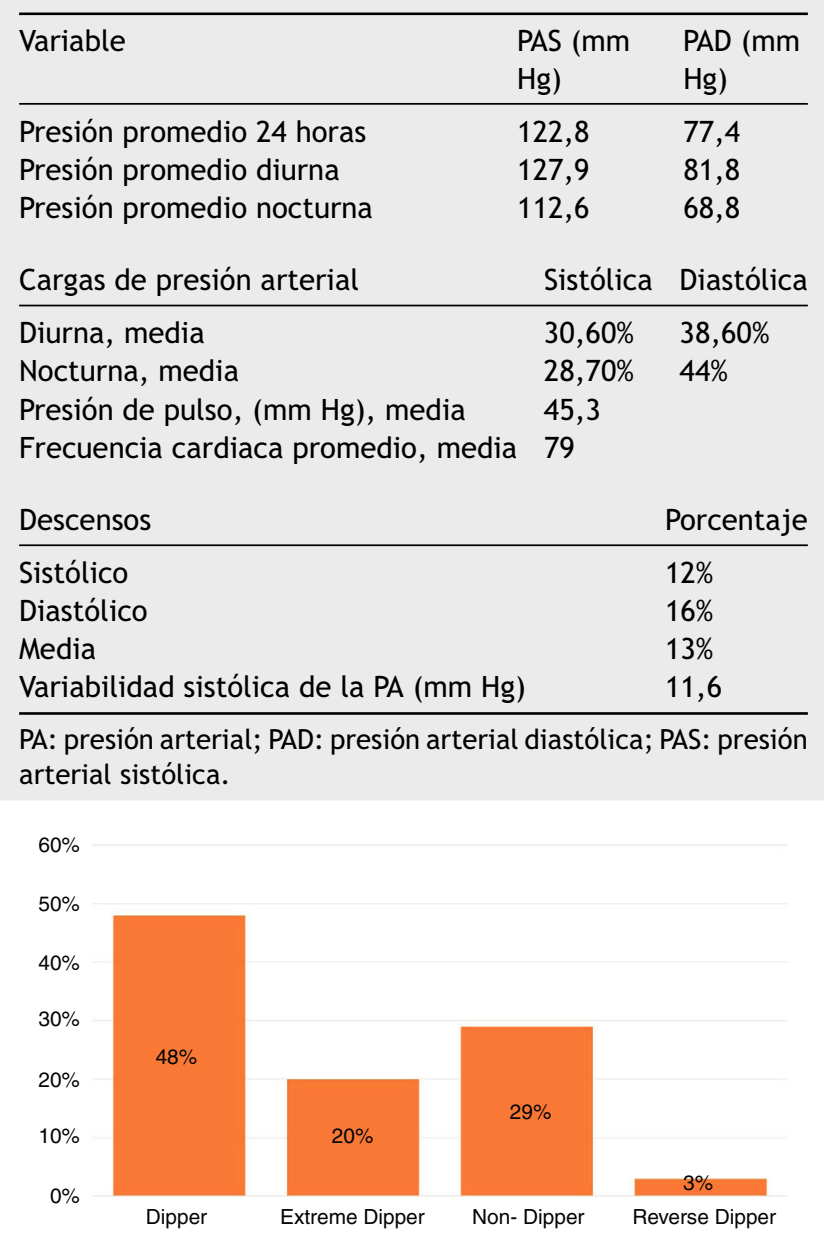

Figura 3 Patrón circadiano de los pacientes del EPEDMAPA.

esfigmomanómetro y estetoscopio, dada su facilidad de uso y bajo costo.

Con el advenimiento de los sistemas automatizados de medición de la presión arterial se han podido establecer muchas otras variables clínicas que contribuyen a un mejor abordaje de los pacientes en cuanto a diagnóstico, direccionamiento de la terapia farmacológica e incluso estableciendo pronóstico ${ }^{7}$.

El registro EPEDMAPA es un esfuerzo por tratar de describir por primera vez en Colombia dicha caracterización clínica, con variables hemodinámicas, patrón circadiano y control de tratamiento de pacientes sometidos a una monitorización ambulatoria de la presión arterial.

Debido a la técnica de medición de presión arterial empleada, el trabajo demostró la existencia de un porcentaje alto de hipertensión arterial nocturna en la población estudiada. Tipificar el predominio horario de la hipertensión arterial sistémica permite identificar grupos poblacionales con mayor riesgo cardiovascular ${ }^{7}$; además, facilita instaurar un plan de tratamiento médico acorde con su perfil de hipertensión.

Es importante resaltar que, entre las medidas farmacológicas instauradas, la mayoría de esta cohorte de pacientes solo requirió el uso de un medicamento antihipertensivo. 
Esta situación permite estimar la importancia del uso de la técnica automatizada de medición de cifras tensionales en cuanto a dirigir el plan terapéutico de los pacientes hipertensos e indirectamente podría reflejar la posibilidad de generar un ahorro en el uso de medicamentos. Sin embargo, para tal conclusión se requieren estudios posteriores que evalúen dicha hipótesis.

El alto porcentaje de pacientes no descendedores nocturnos (reverse dipper y non-dipper) encontrados en este trabajo debe alertar al clínico a tratar de no obviar un posible compromiso orgánico dado el riesgo de asociación con entidades como hipertrofia ventricular, insuficiencia cardiaca, infarto al miocardio, eventos cerebrovasculares y enfermedad renal ${ }^{6,7}$.

El 31\% de los pacientes observados en la cohorte, que previamente habían sido clasificados como hipertensos pero cuyo reporte de monitorización ambulatoria de la presión arterial excluyó la entidad, permite abrir otro punto de discusión acerca de la posible aparición de hipertensión arterial de bata blanca, porcentaje nada despreciable si se tiene en cuenta que se trata de casi un tercio de la población estudiada.

La limitación principal de este trabajo se relaciona con una baja muestra de pacientes en la cohorte. Se abre una brecha importante como línea de investigación para continuar alimentando el registro con más pacientes y eventualmente hacer seguimiento de los mismos a largo plazo, lo que permitiría evaluar otros desenlaces clínicos.

Los resultados del EPEDMAPA deben estimular cada vez más al personal sanitario a tratar de ampliar el uso de la monitorización ambulatoria de la presión arterial como un método de buena práctica clínica.

\section{Conclusiones}

El monitorización ambulatoria de la presión arterial en nuestro medio es una herramienta útil en el diagnóstico y seguimiento de la hipertensión arterial sistémica en concordancia con las actuales guías de manejo para esta enfermedad.

El patrón non-dipper y reverse dipper en conjunto, constituye el $32 \%$ de la población estudiada, situación que debe orientar hacia la búsqueda de causas de hipertensión arterial sistémica subyacentes, potencialmente prevenibles y tratables, como el síndrome de apnea del sueño, entre otras.
Hay mayor porcentaje de control de la hipertensión arterial sistémica en los pacientes cuya terapia fue controlada a través de monitorización ambulatoria de la presión arterial en comparación con los registros existentes controlados por medio de esfigmomanómetro de consultorio.

Se proponen los umbrales de monitorización ambulatoria de la presión arterial de 24 horas como valores para determinar el control de la presión arterial que hoy se han considerado como meta para la definición de hipertensión arterial resistente. Por tanto se requiere evaluación a largo plazo para determinar una correlación con desenlaces clínicos cardiovasculares.

EPEDMAPA es el único registro en Colombia de caracterización clínica, hemodinámica, de variables circadianas y control de tratamiento de pacientes sometidos a monitorización ambulatoria de la presión arterial.

\section{Conflicto de intereses}

Ninguno.

\section{Bibliografía}

1. Kearney PM, Whelton M, Reynolds K, Whelton PK, He J. Worldwide prevalence of hypertension: a systematic review. J Hypertens. 2004;22:11-9.

2. Chobanian AV, Bakris GL, Black HR, Cushman WC, Green LA, Izzo $\mathrm{JL}$, et al. The Seventh Report of the Joint National Committee on Prevention, Detection Evaluation, and Treatment of High Blood Pressure: the JNC 7 report. JAMA. 2003;289:2560-72.

3. II Estudio Nacional de Factores de Riesgo de Enfermedades Crónicas. ENFREC II. Tomo III. Serie de Documentos Técnicos 007. Colombia: Ministerio de Salud; 1999.

4. Hernández-Hernández R, Silva H, Velasco M, Pellegrini F, Macchia A, Escobedo J, et al. Hypertension in seven Latin American cities: the Cardiovascular Risk Factor Multiple Evaluation in Latin America (CARMELA) study. J Hypertens. 2010;28:24-34.

5. Myers MG, Godwin M, Dawes M, Kiss A, Tobe SW, Kaczorowski J. Measurement of Blood Pressure in the Office: Recognizing the Problem and Proposing the Solution. Hypertension. 2010;55:195-200.

6. Pickering TG, Shimbo D, Haas D. Ambulatory Blood-Pressure Monitoring. N Engl J Med. 2006;354:2368-74.

7. O’Brien E, Parati G, Stergiou G, Asmar R, Beilin L, Bilo G, et al. European Society of Hypertension Position Paper on Ambulatory Blood Pressure Monitoring. J Hypertens. 2013;31:1731-68. 